\section{Eine shiny windows theory?} Kommentar zu Ray Forrest, Sin Yee Koh und Bart Wissinks „Hyper-Spaltung in Städten und die ,unmoralischen“ Superreichen“
,Hypergespaltene Städte und die ,unmoralischen' Superreichen - Fünf abschließende Fragen'

Kommentare von: Michael Hartmann, Susanne Heeg, Anna-Lisa Müller, Laura Calbet i Elias

Anna-Lisa Müller

Die Autor_innen Ray Forrest, Sin Yee Koh und Bart Wissink adressieren in ihrem Beitrag ein für die internationale Stadtforschung wichtiges Thema: die aktuell zu beobachtende Tendenz der sozialräumlichen Ausdifferenzierung in Städten, welche mit einer intensiver werdenden sozialen Ungleichheit und räumlichen Fragmentierung einhergeht. Empirischer Gegenstand ihrer Beobachtung ist die sozialräumliche Konzentration extrem reicher Menschen in urbanen Agglomerationen. Die Autor_innen formulieren ihre Beobachtung auf pointierte Weise und skizzieren fünf zentrale Punkte für die Analyse dieses Phänomens: die Herausarbeitung (1) seiner neuen, anderweitig nicht bekannten Merkmale; (2) der mit der Elite der Superreichen verbundenen Infrastrukturen sowie (3) der Politiken der (städtischen) Regierungen, die dazu beitragen, das Phänomen entstehen zu lassen; (4) die Bezugnahme auf Theorien der Neoliberalisierung als Rahmen für die Analyse des Phänomens sowie (5) die Berücksichtigung der lokalen Besonderheiten des global beobachtbaren Phänomens der Konzentration von Superreichen in bestimmten Städten und Stadtteilen.

Ich konzentriere mich in diesem Kommentar auf drei Aspekte: (1) die Frage nach dem Verhältnis von strukturellen, institutionellen und individuellen Handlungen, die auf die grundsätzliche Frage nach der agency von Akteur_innen auf der Makro-, Meso- und Mikroebene verweist und von den Autor_innen als die Frage nach der Rolle der, intermediären Instanzen' gerahmt wird; (2) die auf der Metaebene angesiedelte Frage nach der Bedeutung der Auseinandersetzung mit diesem Phänomen für die Theoriebildung in der (interdisziplinären) Stadtforschung. In beiden Fällen werde ich den Blick insbesondere auf die Bedeutung von Infrastrukturen und Technologien und ihrer Bedeutung für die (Re-)Produktion von gesellschaftlichen und politischen Verhältnissen und Machtstrukturen legen. Darüber hinaus erscheint mir (3) eine Perspektive, die die Autor_innen eher en passant erwähnen, beachtenswert: die Position der Forschenden in der Debatte gesellschaftlicher Phänomene.

Zunächst zu der Konzentration von Superreichen in Städten. Die Autor_innen verweisen besonders auf die institutionelle und strukturelle 
Einbettung dieser städtischen Elite. Sie sei ein Symptom einer umfassenden Transformation des Städtischen, welche durch neoliberale Politiken, Investitionsstrategien unterschiedlicher Akteure und globale sozio-ökonomische Netzwerke erst ins Werden gebracht wird. Ihre sozialräumliche Konzentrationen und die mit ihnen einhergehenden Fragmentierungen ließen sich zudem heute nicht nur in Städten des Globalen Südens finden, sondern ebenso im Globalen Norden. Somit wirkten sie sich auch auf soziale Gruppen der mittleren und oberen Schichten aus - und damit auf diejenigen, die ebenfalls mit einem nicht unbeträchtlichen ökonomischen und sozialen Kapital ausgestattet sind und daher von steigenden Immobilien- und Mietpreisen oder einem angespannten Wohnungsmarkt erst seit kürzerer Zeit merklich betroffen sind. Dies bedeutet allerdings auch, dass heute ein großer Teil der Gesellschaft von der Konzentration dieser - in quantitativer Hinsicht kleinen, aber qualitativ bedeutsamen - Elite in ausgewählten Städten und der daraus entstehenden, global wirksamen sozialen und räumlichen Fragmentierung betroffen ist. Dass dazu nun ein großer Teil der im Diskurs stärker repräsentierten Mitglieder westlich-industrialisierter Gesellschaften gehört, mag die zunehmende (mediale) Aufmerksamkeit zusätzlich erklären. Inwiefern es sich hierbei um eine Fragmentierung im Sinn einer Entkopplung von Teilbereichen handelt, welche fundamentale Folgen insbesondere für die schon Marginalisierten haben kann (zum Beispiel Wacquant 2007), ist eine empirische Frage, die an anderer Stelle zu diskutieren ist.

Die Wirkmächtigkeit der Gruppe der Superreichen erklären die Autor_innen mit ihrer Einbettung in ein Netz aus intermediären Instanzen und Strukturen: „Das Akkumulationssystem der Superreichen [ist] auf ein komplexes Zusammenspiel zwischengeschalteter Institutionen und sich wandelnder struktureller Bedingungen angewiesen.“

Die Autor_innen weisen zu Recht darauf hin, dass es sich bei dem von ihnen adressierten Fall nicht um ein singuläres Phänomen der sozialräumlichen Fragmentierung handelt, sondern die räumliche Konzentration Superreicher ein Ausdruck neben anderen für aktuell zu verzeichnende Ungleichheiten ist. Dies ist ein in dieser Debatte - auch in der Diskussion etwa von Gentrifizierungsphänomenen, die im Kern ebenfalls sozialräumliche Ungleichheitsphänomene darstellen - oft unterrepräsentierter Aspekt. Die Akteure und ihre Beziehungen und Verflechtungen ernst zu nehmen ist, und da stimme ich den Autor_innen zu, wichtig, um die „wachsende[n] urbanenUngleichheiten" mit ihren gesamtgesellschaftlichen Bedingungen und Konsequenzen zu analysieren.

Um eine derartige Perspektive zu realisieren, plädieren die Autor_innen für einen genaueren Blick auf Strukturen und dabei insbesondere auf die intermediären Akteure, etwa lokale Regierungen und Politiken. Dies ist für die sozialwissenschaftliche Analyse, die Aussagen über städtische und gesellschaftliche Prozesse treffen will, besonders interessant, da dadurch der Blick auf das ,größere Ganze' gerichtet wird und nicht ausschließlich individuelle Personen für Entwicklungen verantwortlich gemacht werden, die sie nicht alleine verantworten. Vielmehr sind sie und ihre Anwesenheit in Städten in strukturelle, institutionelle, historische und lokal spezifische Entwicklungen eingebettet, deren Konsequenzen nicht nur eine Fragmentierung von Städten entlang ökonomischer Merkmale beinhalten, sondern auch, und darauf 
hinzuweisen verzichten die Autor_innen, in soziale, kulturelle und ökologische Entwicklungen: die Konzentration von sozialen und technologischen Innovationen in urbanen Clustern wie dem Silicon Valley, die selektive ökonomische Vernetzung in Global Cities, die Verortungen global agierender internationaler Organisationen an ausgewählten Standorten insbesondere des Globalen Nordens oder die Verdichtung der Folgen des Klimawandels in Ländern des Globalen Südens.

All dies sind Beispiele für global auffindbare, lokal spezifische Ungleichheiten, die an strukturell (re-)produzierte, oft historisch gewachsene Machtverhältnisse gekoppelt sind und in einer räumlich und sozial vernetzten Welt vielfältige Neben- und Folgeeffekte aufweisen.

Natürlich entsteht mit dieser Fokussierung der Makro- und Mesoebene der Strukturen und Institutionen die Frage nach den individuellen Praktiken und damit nach der Mikro-Ebene. Diese fangen dieAutor_innen insofern ein, als sie die Bedeutung der lokalen Spezifizität der Phänomene hervorheben. Dies ist eine wichtige Ergänzung, die in einem Plädoyer für komparative Forschung kulminiert, da sich das Phänomen als eines zeigt (und auch von den Autor_innen so beschrieben wird), das sich bei globaler Ähnlichkeit je nach lokaler Situiertheit unterschiedlich ausbildet.

Hier ist, so meine ich, insbesondere auf die politischen Rahmenbedingungen, Planungskulturen, Politiken, Institutionen und Gesellschaftssysteme hinzuweisen, welche die lokalen Verhältnisse mit hervorrufen. Hinzu kommen die (im weitesten Sinn lokal, für die Planung auch national) spezifischen Technologien und Infrastrukturen, in die die Institutionen eingebettet sind und die diese stabilisieren; über sie werden befördernde oder hindernde Entwicklungen ebenso unterstützt wie Machtstrukturen und politische Rahmenbedingungen, die bestimmte Verhältnisse mit hervorbringen, aber auch verhindern können. So zeigen Jochen Monstadt und Annika Wolff (2017), dass (technologische) Infrastrukturen in der Geschichte der modernen Stadt immer auch Motor gesellschaftlicher Entwicklungen und damit sozialen Wandels waren, sie aber im Sinn von Infrastrukturregimen spezifische technische, ökonomische und politische Strukturen beinhalten, die Veränderungen sowohl von Politiken als auch von gesellschaftlichen Strukturen ver- oder zumindest behindern können (Monstadt/Wolff 2017: 205ff.). Dies lässt sich für die Infrastrukturen der Superreichen entsprechend weiterdenken. So können etwa die architektonisch vom Rest der Stadt abgeschlossenen Wohnquartiere der Superreichen in Zentrumsnähe die Entstehung öffentlicher Räume materiell und rechtlich verhindern, da der Zugang zu ihnen nicht nur de jure reglementiert, sondern auch physisch erschwert ist. Weniger Gelegenheitsstrukturen zu Begegnungen zwischen heterogenen Gruppen, verbunden mit Irritationen, Konflikt, aber auch Konsensbildung und Anregung, können so zu einer Veränderung des städtischen Zusammenlebens zugunsten eines sozialen und physisch-räumlichen Nebeneinanders und zu einer Manifestierung eines status quo führen: Das Sehen des Bekannten in dem mir zugänglichen Quartier verstärkt seine Beharrungseffekte und erschwert Innovation und Veränderung.

Insgesamt haben wir es daher mit einem interessanten Wechselspiel von Struktur und agency von Sozialität und Materialität zu tun, welches mit Beharrungseffekten und Wandel zusammengeht. Dieses Wechselspiel bleibt 
bei den Autor_innen vergleichsweise unausgearbeitet; die agency, die sie in den Blick nehmen, liegt insbesondere bei den Intermediären, während die strukturellen Bedingungen in erster Linie durch die von ihnen konstatierten neoliberalen Bedingungen und dem damit verbundenen Zusammenspiel von Finanzialisierung und Urbanisierung gebildet werden. Hier wäre es interessant, die in neoliberalen Entwicklungen eingelassenen Paradoxien genauer in den Blick zu nehmen und das Phänomen der fragmentierten Städte entlang sozio-ökonomischer Linien daraufhin zu befragen, welche kollektiven Akteure neben den Investor_innen, Regierungen und Planer_innen eine Rolle bei der (De-)Stabilisierung dieser gesellschaftlichen Verhältnisse spielen.

Indem Stabilisierung und Destabilisierung und damit Stillstand und Dynamik berücksichtigt werden, geraten dann auch die spezifischen (zeitlichen) Merkmale von Entwicklungen, die beispielsweise die Realisierung bestimmter Bauprojekte rahmen, in den Blick der Analyse. So ist es zum Teil erstaunlich, dass bestimmte Projekte etwa im Sozialwohnungsbau einen sehr langen zeitlichen Vorlauf bis zu ihrer Realisierung aufweisen, während andere Entwicklungen - Umwandlung von Stadtquartieren in Orte der Superreichen, zum Teil ,bought to rent ${ }^{*}$ - schneller und in gewisser Hinsicht konfliktfreier realisierbar zu sein scheinen. Dies kann zum einen darüber erklärt werden, dass in vielen demokratischen Gesellschaften die Planungsprojekte für die Allgemeinheit in ein komplexes System aus Entscheidungsstrukturen eingebettet sind. In diesem sollen Beteiligungsverfahren, politische Verhandlungen und Ausschreibungen einerseits in einem utilitaristischen Sinn den größtmöglichen Nutzen für die größtmögliche Zahl an Personen sichern und andererseits dazu beitragen, dass sich die Planungsverfahren über einen langen Zeitraum strecken. Wenn etwa privatwirtschaftliche Investoren ein kleinskaliertes Bauprojekt für die Superreichen planen und realisieren, sind die zu berücksichtigenden Interessen die einer vergleichsweise kleinen und homogenen Gruppe - anders als dies bei Projekten, die das Gemeinwohl betreffen, der Fall ist. Aufgrund der dadurch geringer ausgeprägten Notwendigkeit der Konsensbildung können die Realisierungszeiten derartiger Projekte deutlich geringer sein als in gemeinwohlorientierten Projekten.

Zum anderen lässt sich für die spezifischen Regierungsformen (zum Beispiel Foucault 2009: 151-160) eine je charakteristische Honorierung bestimmter Projektformen und -arten identifizieren, die dazu führt, dass bestimmte politische Projekte in bestimmten Regimen unterschiedlich einfach oder schwer realisiert werden können. Folgt man der Argumentation der Autor_innen, dass wir es gegenwärtig mit einer neoliberalen, durch die Finanzwirtschaft geprägten Logik zu tun haben, die weltweit Gesellschaften und ihre Regierungen prägt, so lässt sich daraus die Vermutung ableiten, dass das Bauen für die Superreichen, insbesondere mithilfe von privaten Investor_innen, die große Teile der Finanzierung übernehmen, im Neoliberalismus eine geförderte, geradezu hegemoniale Planungsform darstellt. In dieser wird demnach etwa der Sozialwohnungsbau als überkommene Form der Sorge des Staates für die Einzelnen interpretiert und daher als randständiges Thema der (politischen) Planung behandelt.

Um das Phänomen in seiner Komplexität angemessen analysieren zu können, brauche es, so die Autor_innen, allerdings auch eine Neufassung vorhandener Sozialtheorien. Ihre eigene Frage: „Was sagt uns dies schließlich 
über Theorien des Urbanen und den Grad ihrer Brauchbarkeit und globalen Anwendbarkeit?" lässt sie konstatieren, dass das vorhandene Theorieangebot nicht ausreichend sei, und einen Ausweg formulieren: „A postcolonial sensibility to urban theory that pays attention to the relationship between place, knowledge, and power" is the way forward." (Roy 2016: 200, Herv. i. Orig.)

Die Beobachtung einer sozialräumlichen Konzentration einer ökonomischen Elite sowohl im Globalen Süden als auch im Globalen Norden ist für die Stadtforschung nicht nur inhaltlich interessant, sondern auch konzeptionell, fordert sie doch zu einer Auseinandersetzung mit vorhandenen, häufig entweder für den Globalen Norden oder den Globalen Süden entwickelten Theorien auf. Wie diese Integration von Theorien geschehen kann, lässt sich an aktuellen Arbeiten zu (städtischen) Infrastrukturen beobachten. Nadine Marquardt (2017) etwa zeigt mithilfe ihrer empirischen Forschung zu Obdachlosen im städtischen Raum, wie Theorien aus dem Kontext des Globalen Südens für eine Analyse von Phänomenen des Globalen Nordens produktiv gemacht werden können. Marquardt (2017: 101) argumentiert, dass, anders als im Globalen Norden, das Funktionieren der Infrastrukturen im Globalen Süden einen ,Sonderfall' darstelle und die kreative Aneignung und Umnutzung von Infrastrukturen der eigentliche Normalzustand sei (vgl. Star (1999) zum Verständnis des Funktionierens von Infrastruktur als ,Normalfall'). Mithilfe dieser von Theorien des Globalen Südens inspirierten Perspektive auf die Nutzer_innen von Infrastrukturen als Ko-Produzent_innen nimmt Marquardt eine Analyse des Umgangs von Obdachlosen mit Infrastrukturen vor und zeigt die daraus entstehenden selbstorganisierten Infrastrukturen im städtischen Raum sowie die spezifischen Formen der sozialen Exklusion/Inklusion, die über infrastrukturelle Ent-/Kopplung entstehen (Marquardt 2017: 94).

An diesem Beispiel zeigt sich, welche theoretisch-konzeptionellen Möglichkeiten die programmatisch formulierte Analyse des von den Autor_innen beschriebenen Phänomens impliziert: Anstatt die sozialräumlichen Wirkungen der in Städten lebenden Superreichen als statisches Phänomen zu untersuchen, könnte durch die Fokussierung ihrer Praktiken sowie ihrer strukturellen Einbettung und die Bedeutung intermediärer Akteure das Gefüge einer Stadt gezeichnet werden, in dem auch die Superreichen als Ko-Produzent_innen agieren und ihre Praktiken in einem von Strukturen und Intermediären abgesteckten Rahmen vollziehen. Dieser Rahmen wird durch ihr Handeln ebenso wie durch das Handeln anderer individueller, kollektiver, institutioneller Akteure aber gerade auch erweitert, verschoben oder modifiziert.

Aus einer Materialitäts- und Infrastruktur-sensitiven Perspektive wäre es daher interessant, zukünftig einen Blick auf die Beharrungseffekte der spezifischen, für die Elite der Superreichen entwickelten sozialen und physischen Infrastrukturen zu richten. Folgt man der Annahme, dass gebaute Strukturen den in ihnen verorteten Institutionen eine zusätzliche zeitliche Beständigkeit verleihen (zum Beispiel Hirst 2005), so wirft das für die Zukunft der physisch-räumlichen, aber auch sozialen Gestaltung von Städten, die durch die Existenz der Superreichen geprägt wird, interessante Forschungsfragen auf. Wenn Thomas F. Gieryn (2002) am Beispiel des Neubaus eines Universitätsgebäudes zeigt, wie dieses die Entstehung und 
Ausformung einer wissenschaftlichen Disziplin mit beeinflusst, so ließe sich dies auch für die Gesellschaft von Städten und für ihre Institutionen zeigen: Sind bestimmte Infrastrukturen, etwa die privaten Clubs und Meerzugänge, die im Sammelband von Forrest et al. besprochen werden, erst einmal installiert und werden durch ihre Nutzung in die alltäglichen Praktiken der Menschen (der Clubmitglieder, der Angestellten, der Besitzer_innen) inkorporiert, können sie ähnliche Beharrungseffekte aufweisen wie im anderen Fall die Stigmatisierungen von Quartieren und dort lebenden Menschen über die nicht vorhandene oder schlechte Infrastruktur. Häußermann und Siebels (2004: 159) Beschreibung der Entwicklung eines „benachteiligten Quartiers“ zu einem „benachteiligenden Quartier“ ließe sich in diesem Sinn also möglicherweise - und dies ist die offene empirische Frage - dahingehend wenden, dass ein infrastrukturell und technologisch bevorteiltes Quartier nicht nur zu einem sozial und politisch bevorteilenden Quartier wird, sondern dies auch dann noch bleibt, wenn die Superreichen selbst schon an andere Orte gezogen sind oder von anderen hegemonialen Gruppen abgelöst wurden. Bezugnehmend auf die broken windows theory (Wilson/ Kelling 1982) und die Beobachtungen und konzeptionellen Forderungen von Forrest et al. weiterdenkend ließe sich als Modell eine shiny windows theory denken: eine Theorie, die auf die Wirksamkeit und zeitliche Beständigkeit von (materiellen) Bedingungen in ihrem gebauten Glanz (und nicht ihrer Zerstörung) verweist und helfen könnte, die Beharrungseffekte, die diese haben können, für die Konstitution von Gesellschaft zu erklären.

Mein dritter Kommentar adressiert schließlich unsere Selbstreflexivität als Wissenschaftler_innen, da das programmatische Anliegen der Autor_innen nicht nur die Beobachtung beinhaltet, dass es eine sozialräumliche Konzentration von Superreichen gibt. Vielmehr sei ,in der Populärkultur wie auch in weiten Teilen der akademischen Literatur eine deutliche Tendenz [zu konstatieren], eine neue superreiche Elite für die dramatischen, von gröBeren sozialen und räumlichen Ungleichheiten geprägten Veränderungen der urbanen Morphologie verantwortlich zu machen“. Diese Beobachtung, die (auch) das Arbeiten der Forschenden betrifft, gilt, so möchte ich argumentieren, nicht nur für das hier behandelte Phänomen der Superreichen in Städten. Wir haben es vielmehr mit einem Beispiel für ein grundlegenderes Phänomen zu tun, das auch die (Sozial-)Wissenschaft im Kern angeht und ihre Arbeitsweise und Forschungsthemen betrifft. Ähnlich wie im Fall von Gentrifzierungsphänomenen findet fallweise vor oder parallel zu einer fundierten empirischen Analyse und ihrer theoretisch-konzeptionellen Interpretation eine selten explizit als solche dargestellte normative Rahmung des Untersuchungsgegenstandes statt. Diese normative Rahmung geht häufig mit einer Vorverurteilung individueller und institutioneller Handlungsweisen und -muster einher, welche verhindert, dass die wissenschaftliche und öffentliche Debatte über gesellschaftliche Phänomene konstruktiv erhellt wird.

Natürlich sind die Forschenden, und dies gilt insbesondere für Sozialwissenschaftler_innen, immer auch über ihre individuellen und kollektiven Erfahrungen als Forschende und als Gesellschaftsmitglieder Teil der Phänomene, die sie untersuchen und die sie zu verstehen und erklären trachten (vgl. Reuter/Villa 2010: 23; dazu auch aus dezidiert feministischer Perspektive Harding 1991, Haraway 1995). Im Sinn einer den Leitideen der 
qualitativen Forschung nahestehenden Forschungshaltung, die die einzelnen Gesellschaftsmitglieder mit ihren kollektiven „Sinnzuweisungen“, „Sinnkonstitutionen“ sowie ihrem „Sinnverstehen“ ernst nimmt (zum Beispiel Lamnek 1995: 25), ist es allerdings erforderlich, diese subjektiven und situativ spezifischen Prozesse in ihrer Komplexität nachzuzeichnen und zu kontextualisieren. Erst dann kann, eine entsprechende Intention der Forschenden vorausgesetzt, eine Bewertung des Phänomens und eine (normative) Positionierung der Forschenden zum Phänomen stattfinden - wie sie dieAutor_innen am Ende ihres Textes auch einfordern: „Wir sollten uns auch weiterhin über die eklatanten und wachsenden Ungleichheiten in unseren Städten empören“. Dies kann, neben der Analyse und Theorieentwicklung, unsere Aufgabe sein, zu der Forrest, Koh und Wissink einen anregenden Vorstoß formuliert haben.

Die Publikation dieses Beitrags wurdevon der Universität Bremen gefördert.

\section{Autor innen}

Anna-Lisa Müller ist Humangeographin und Stadtsoziologin. Sie arbeitet zu den Themen Stadt, Migration und qualitative Methoden der Sozialforschung.

anna-lisa.mueller@uni-bremen.de

\section{Literatur}

Foucault, Michel (2009): Sicherheit, Territorium, Bevölkerung. Geschichte der Gouvernementalität I. Bd. 1. Vorlesung am Collège de France 1977/1978. Frankfurt am Main: Suhrkamp.

Gieryn, Thomas F. (200). What buildings do. In: Theory and Society 31/1, 35-74.

Haraway, Donna (1995): Situiertes Wissen. Die Wissenschaftsfrage im Feminismus und das Privileg einer partialen Perspektive. In: Carmen Hammer / Immanuel Stieß (Hg.), Die Neuerfindung der Natur. Primaten, Cyborgs und Frauen. Frankfurt am Main: Campus, 73-97.

Harding, Sandra G. (1991): Whose science? Whose knowledge? Thinking from women's lives. Ithaca: Cornell University Press.

Häußermann, Hartmut / Siebel, Walter (2004): Stadtsoziologie. Eine Einführung. Frankfurt am Main/New York: Campus.

Hirst, Paul (2005): Space and Power: Politics, War and Architecture. Malden/MA: Blackwell.

Lamnek, Siegfried (1995): Qualitative Sozialforschung, Bd.1: Methodologie. Weinheim: Beltz.

Marquardt, Nadine (2017): Zonen infrastruktureller Entkopplung. Urbane Prekarität und soziotechnische Verknüpfung im öffentlichen Raum. In Michael Flitner / Julia Lossau / Anna-Lisa Müller (Hg.), Infrastrukturen der Stadt. Wiesbaden: VS, 89-104.

Monstadt, Jochen / Wolff, Annika (2017): Infrastrukturregime und inkrementeller Wandel. In Michael Flitner / Julia Lossau / Anna-Lisa Mlller (Hg.), Infrastrukturen der Stadt. Wiesbaden: VS, 205-225.

Reuter, Julia / Villa, Paula-Irene (2010): Provincializing Soziologie. Postkoloniale Theorie als Herausforderung. In Julia Reuter / Paula-Irene Villa (Hg.), Postkoloniale Soziologie. Empirische Befunde, theoretische Anschlüsse, politische Intervention. Bielefeld: transcript, 11-46.

Roy, Ananya (2016): Who's Afraid of Postcolonial Theory? In: International Journal of Urban and Regional Research 40/1, 200-209.

Star, Susan Leigh (1999): The Ethnography of Infrastructure. In: American Behavioral Scientist 43/3, 377-91. 
Wacquant, Loic (2007): Territorial stigmatization in the age of advanced marginality. In: Thesis Eleven 91/1, 66-77.

Wilson, James Q. / Kelling, George E. (1982): Broken windows. The police and neighboorhood safety. In: The Atlantic Monthly 249/3, 29-38. 